\title{
LEIOMIOSSARCOMA DO ESÔFAGO
}

\section{ESOPHAGEAL LEIOMYOSARCOMA}

\section{Geraldo José Souza Nascimento, ACBC-BA ${ }^{1}$; Claudio de Almeida Quadros, ACBC-BA ${ }^{2}$; Carlos Eduardo Pinto, TCBC-RJ ${ }^{3}$}

\section{INTRODUÇÃO}

O leimiossarcoma do esôfago representa uma rara enfermidade, responsável por cerca de 0,5 a $1,0 \%$ das neoplasias esofágicas. Apesar disto é considerado o mais comum sarcoma primário do esôfago, embora muito menos freqüente que os sarcomas de outros locais do trato gastrointestinal (estômago e intestino delgado).

\section{RELATO DO CASO}

N.R.B., 45 anos, feminina, referia disfagia para sólidos. A esofagografia mostrava falha de enchimento ao nível da JEG, regular, com dilatação à montante, sugerindo não haver comprometimento da mucosa. Na TC do tórax, evidenciava-se volumosa massa em mediastino posterior, que deslocava a aorta para trás e estendia-se para o abdome (Figura 1). A TC do abdome mostrava espessamento do esôfago terminal e do estômago proximal. A EDA identificava, aos $35 \mathrm{~cm}$ dos incisivos, grande protusão da mucosa, ligeiramente avermelhada, íntegra, sugerindo compressão extrínseca ao nível do esôfago distal, compatível com leiomioma.

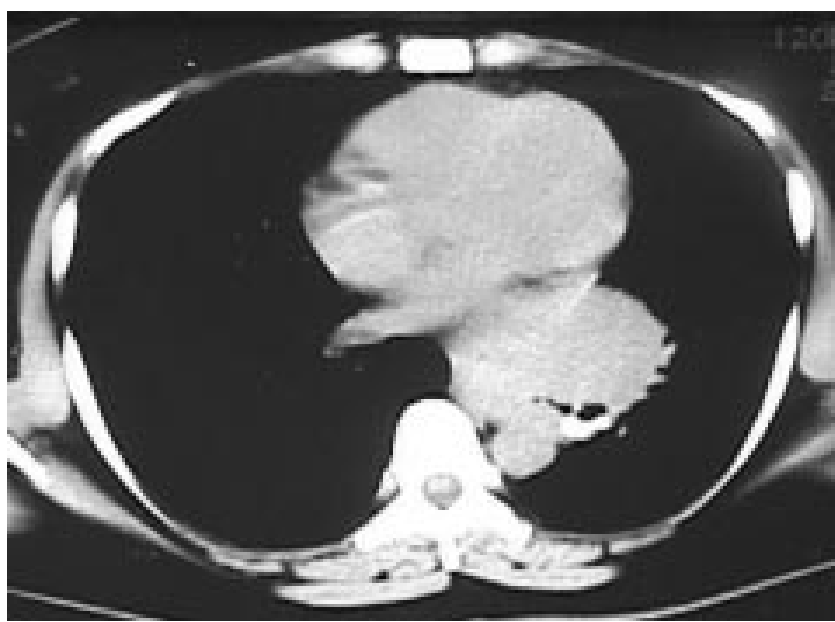

Figura 1 - CT de Tórax evidenciando tumoração no mediastino posterior.
A paciente foi submetida à esofagectomia, com reconstrução do trânsito utilizando tubo gástrico através do mediastino posterior (Figura 2). O tempo cirúrgico foi de 6 h,e não houve hemotransfusão. Evoluiu sem complicações, com alta no $13^{\circ}$ dia de pós-operatório, aceitando bem dieta por via oral. O exame histopatológico e a imuno-histoquímica confirmaram tratar-se de leiomiossarcoma do esôfago. Atualmente encontra-se no $7^{\circ}$ ano de seguimento, sem recidiva e sem disfagia, com ganho ponderal de $8 \mathrm{Kg}$.

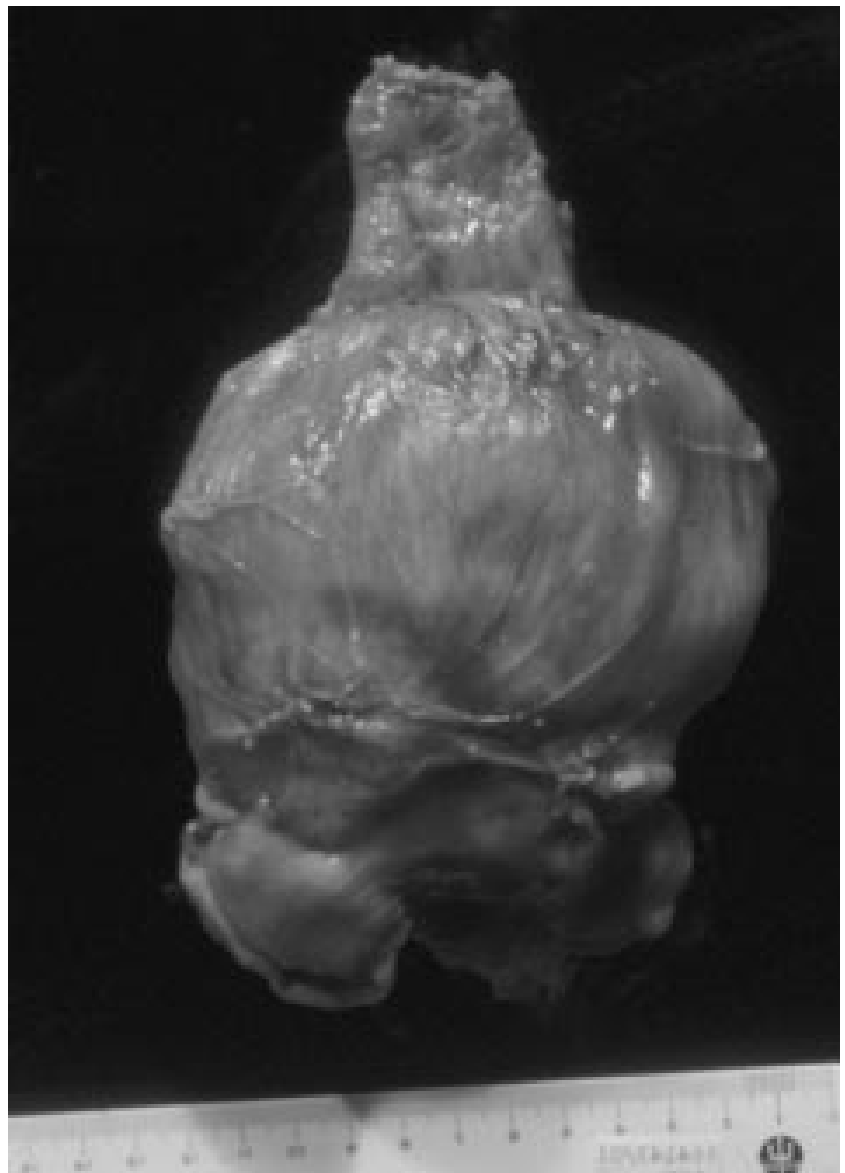

Figura 2 - Peça cirúrgica constituída de esofagectomia com leiomiossarcoma de esôfago inferior.

1. Cirurgião Oncológico do Centro de Hematologia e Oncologia - CEHON, Hospital São Rafael e Hospital Salvador; Chefe da Sessão de Oncologia do Hospital Geral do Exército - 6 RM, Salvador - Bahia

2. Cirurgião Oncológico da Sociedade de Oncologia da Bahia - ONCO e Hospital Salvador; Chefe do Serviço de Coloproctologia do Hospital Aristides Maltez, Salvador - Bahia.

3. Coordenador do Grupo de Esôfago do Serviço de Cirurgia Abdomino-pélvica do INCA; Doutor e Mestre em Cirurgia pela UFRJ.

Recebido em 09/04/2004

Aceito para publicação em 19/11/2004

Trabalho realizado no Serviço de Cirurgia Abdômino-pélvica do Instituto Nacional do Câncer - INCA, Rio de Janeiro - RJ. 


\section{DISCUSSÃo}

Apesar de incomuns, os tumores originários da musculatura lisa são as neoplasias mesenquimais mais freqüentes do esôfago ${ }^{1}$. Os sarcomas primários do esôfago são ainda mais raros, representando cerca de 0,5 a $1 \%$ das neoplasias esofágicas ${ }^{1}$. Até 1999 apenas 100 casos tinham sido relatados na literatura japonesa ${ }^{2}$.

Os leiomiomas são os tumores submucosos mais comuns do esôfago e seis vezes mais freqüentes que os leiomiossarcomas, sendo o diagnóstico diferencial pré-operatório extremamente difícil. Apenas em 15 a $40 \%$ dos casos é possível realizar o diagnóstico antes da cirurgia ${ }^{3,4}$. Com o objetivo de melhorar esse percentual, alguns critérios têm sido utilizados: 1) tamanho do tumor; 2) presença de ulceração; 3) massa heterogênea ou com áreas de baixa densidade central à tomografia computadorizada $;$ 4) ultra-sonografia endoscópica demonstrando irregularidades internas e ecos periféricos; 5) hipervascularização à angiografia. Apesar de haver boa correlação desses critérios com o diagnóstico final, o estudo anatomopatológico permanece como exame de primeira linha para confirmação.

Clinicamente é caracterizado pelo seu curso clínico indolente, tendo a disfagia como sintoma principal. Alguns pacientes são assintomáticos, e o diagnóstico é feito em exames de rotina ${ }^{1}$.

Habitualmente localizam-se no terço médio e inferior do esôfago, onde há predominância da musculatura lisa e em $60 \%$ das vezes apresentam-se sob a forma polipóide. Achados radiológicos do leiomiossarcoma primário do esôfago são semelhantes aos dos sarcomas primários de outros órgãos do trato gastrointestinal. ${ }^{3} \mathrm{~A}$ presença de ulcerações pode levar o endoscopista a confundir o leiomiossarcoma com o carcinoma epidermóide. Estando a mucosa íntegra, a realização de biópsias é de pouca utilidade pela característica superficial do fragmento retirado, devido à natureza submucosa destas lesões. O surgimento da ultra-sonografia endoscópica possibilitou a determinação de critérios específicos para o diagnóstico destas lesões, tais como: tumor bem definido, homogêneos e hipoecóico com fartos ecos marginais originados na camada muscular do esôfago.

Apesar de metástases serem infrequentes, a sobrevida em cinco anos é de 35 a $50 \% .^{1}$ Devido à dificuldade de definição pré-operatória entre leiomiossarcoma e leiomioma, a abordagem cirúrgica com radicalidade oncológica é o tratamento de escolha.

\begin{abstract}
The authors describe a case of esophageal leiomyosarcoma treated at the Abdominopelvic Surgery Department of the Brazilian National Cancer Institute, including literature review, addressing treatment and prognosis. A 45 year-old female patient complaining of dysphagia, with pre-operative exams sugestive of esophageal leiomyoma. She was submitted to an esophagusgastrectomy with digestive reconstitution using a gastric tube brought through the posterior mediastinum. The histopathological examination and immunohistochemical tests confirmed that the tumor was an esophageal leiomyosarcoma. She is at the $7^{\text {th }}$ year of follow up with no recurrence nor digestive complains (Rev. Col. Bras. Cir. 2005; 32(4): 218-219).
\end{abstract}

Key Words: Esophagus; Leiomyosarcoma; Esophageal neoplasms.

\section{REFERÊNCIAS}

1. Aiko S, Yoshizumi Y, Sugiura Y, et al. Pedunculated esophageal leiomyosarcoma: a case report. Dis Esophagus. 1998; 11(4):263-7.

2. Athanasoulis CA, Aral IM. Leiomyossarcoma of the esophagus. Gastroenterology. 1968; 54(2):271-4.

3. Levine MS, Buck JL, Pantongrag-Brown L, et al. Leiomysarcoma of the esophagus: radiolografc findings in 10 patients. AJR Am J Roentgenol. 1996; 167(1):27-32.
4. Gray SW, Skandalakis JE, Shephard D. Smooth muscle tumors of the esophagus. Int Abstr Surg. 1961; 3:205-20.

5. Clark RA, Alexander ES. Computed tomography of gastrointestinal leiomyosarcoma. Gastrointest Radiol. 1982; 7(2):127-9.

Endereço para correspondência:

Dr. Geraldo José Souza Nascimento

Cond. Rio das Pedras; Ed. Safira; APT 701; Imbuí

41720-050 - Salvador - Bahia

e-mail: geraldo.n@terra.com.br 